1994

\title{
Impact of Field-Dependent Electronic Trapping Across Coulomb Repulsive Potentials on Low Frequency Charge Oscillations
}

\author{
R. P. Joshi \\ Old Dominion University \\ K. H. Schoenbach \\ Old Dominion University \\ P. K. Raha \\ Old Dominion University
}

Follow this and additional works at: https://digitalcommons.odu.edu/bioelectrics_pubs

Part of the Electrical and Electronics Commons, and the Probability Commons

\section{Repository Citation}

Joshi, R. P.; Schoenbach, K. H.; and Raha, P. K., "Impact of Field-Dependent Electronic Trapping Across Coulomb Repulsive Potentials on Low Frequency Charge Oscillations" (1994). Bioelectrics Publications. 245.

https://digitalcommons.odu.edu/bioelectrics_pubs/245

\section{Original Publication Citation}

Joshi, R. P., Schoenbach, K. H., \& Raha, P. K. (1994). Impact of field-dependent electronic trapping across Coulomb repulsive potentials on low frequency charge oscillations. Journal of Applied Physics, 75(8), 4016-4021. doi:10.1063/1.356024

This Article is brought to you for free and open access by the Frank Reidy Research Center for Bioelectrics at ODU Digital Commons. It has been accepted for inclusion in Bioelectrics Publications by an authorized administrator of ODU Digital Commons. For more information, please contact digitalcommons@odu.edu. 


\title{
Impact of field-dependent electronic trapping across Coulomb repulsive potentials on low frequency charge oscillations
}

\author{
R. P. Joshi, K. H. Schoenbach, and P. K. Raha \\ Department of Electrical and Computer Engineering, Old Dominion University, Norfolk, \\ Virginia 23529-0246
}

(Received 19 October 1993; accepted for publication 15 December 1993)

\begin{abstract}
We have performed Monte Carlo simulations to obtain the field dependence of electronic trapping across repulsive potentials in GaAs. Such repulsive centers are associated with deep level impurities having multiply charged states. Our results reveal a field-dependent maxima in the electronic capture coefficient, and the overall shape is seen to depend on the background electron density due to the effects of screening. Based on the Monte Carlo calculations, we have examined the stability of compensated semiconductors containing such repulsive centers. Our analysis indicates a potential for low frequency charge oscillations which is in keeping with available experimental data.
\end{abstract}

\section{INTRODUCTION}

Trapping of mobile carriers at defect levels lying within the forbidden gap of semiconductors is an important process. It enhances the turn-off characteristics of electronic devices by providing efficient carrier removal through indirect recombination. ${ }^{1}$ Traps also assist electron tunneling (both elastic and inelastic), and can contribute to current transport across potential barriers. In addition, defects dictate the emission characteristics of light-emitting diodes, affect quantum efficiencies of photodetectors, and degrade the transistor gain parameters. Furthermore, freecarrier trapping is known to produce current fluctuations $\mathrm{s}^{2,3}$ and to directly influence the noise spectral density at low electric fields. ${ }^{4,5}$ Transport in the presence of carrier trapping is strongly modified due to space-charge effects associated with the formation of localized high field regions. ${ }^{6,7}$ A highly nonlinear electrical behavior results from the interplay of nonlocal impact ionization, field-enhanced emission, and satellite valley transfers.

An electric field dependence of the electron trapping phenomena can lead to the formation of domains and cause current oscillations in semiconductor materials. As first demonstrated by Ridley, ${ }^{8}$ low- and high-field domains can develop if the capture coefficient increases with the electric field. This was later supported by the work of Sacks and Milnes. ${ }^{9}$ However, such field-dependent enhancements of the capture coefficient are possible only if the trapping process entails a repulsive potential barrier. This occurs because in such a situation, an applied external field increases the average kinetic energy of the electrons, making it easier for them to surmount the potential barrier. Furthermore, trapping at attractive Coulomb centers could not produce the desired electric field dependence, since the field-induced carrier heating would only decrease the capture probability. This would ensue because a longer sequence of phonon emissions would be required for the eventual capture of mobile electrons. Direct experimental data validating the above argument for attractive potentials exists, ${ }^{10}$ and quantitative Monte Carlo simulations of the field-dependent behavior in GaAs have also been carried out. ${ }^{11}$ From the standpoint of trapping-controlled current oscillations, therefore, the more commonly studied attractive Coulomb centers are not as interesting. Instead, one needs to examine the role of repulsive defects.

Despite the importance of free carrier trapping and detrapping, microscopic calculations pertaining to repulsive potential barriers have not been performed to the best of our knowledge. The dynamics of charge trapping and capture at attractive Coulomb centers, on the other hand, have been well studied in the past. For example, calculations based on the multiphonon cascade model of $\mathrm{Lax}^{12}$ have yielded good results with experimental findings. ${ }^{13} \mathrm{~A}$ number of modifications and extensions of the basic theory have also been developed. ${ }^{14,15}$ More recently, microscopic simulations for electronic trapping at attractive impurity centers in $\mathrm{GaAs}$ were performed ${ }^{11}$ based on a numerical Monte Carlo procedure. ${ }^{16}$ Such calculations for the detailed trapping dynamics have explicitly taken account of free carrier screening effects, the role of carrier-carrier scattering, and included the electric field dependence.

The phenomena of detrapping and impact ionization at a trapping center, which constitutes the reverse process, has also been well investigated at the microscopic level. Shichijo and Hess, ${ }^{17}$ for example, performed Monte Carlo studies of the intrinsic and extrinsic impact ionization processes due to both electrons and holes. A full band structure was employed for accurate and meaningful calculations. Similarly, the detrapping dynamics in the context of high-field transport in $\mathrm{SiO}_{2}$ was analyzed on the basis of a microscopic model which coupled rate equations with a Monte Carlo approach. ${ }^{18}$ However, a similar evaluation of electronic trapping across repulsive potentials remains an unresolved issue.

Charged centers formed due to the presence of impurities and defects, can often be multiply ionized. For example, copper, zinc, and iron are some of the more familiar deep level impurities which can exist in $\mathrm{Si}, \mathrm{Ge}$, or $\mathrm{GaAs}{ }^{19}$ These impurities exhibit multiple charged states as has been observed from photo-Hall measurements, and constitute repulsive trapping centers that are of interest in the present context. Such impurity centers in semiconducting 
material are technologically important for a variety of reasons. For example, they provide the means of fabricating highly resistive substrates, lead to improved electrical device isolation in integrated circuits, afford a very useful base material for optically controlled high power repetitive switches, ${ }^{20}$ and can be used to efficiently generate terahertz radiation. ${ }^{21}$

In this work, we perform semiclassical Monte Carlo simulations to obtain the electric-field dependence of the electronic capture process across repulsive barriers. Such potentials, as stated earlier, could result from the presence of deep impurities in semi-insulating material. Based on the field-dependent capture cross-sections obtained, we evaluate the stability of a semiconductor system containing such multiply ionized trap states. It must be emphasized, however, that the present work focuses only on the trapping process as a possible source of instabilities in semiinsulating semiconductors, but does not preclude the role of other mechanisms. For example, nonlocal impact ionization, ${ }^{22}$ two-stream instabilities and optical phonon emission, ${ }^{23}$ and detrapping within high-field domains ${ }^{7}$ have also been shown to be potential sources for current oscillations and instabilities.

\section{SIMULATION PROCEDURE}

We primarily focus on the role of negatively charged impurities in semi-insulating semiconductors, though trapping at potential barriers across heterointerfaces can also be treated within the general framework of our simulation. Negatively charged impurity centers afford an easier treatment since an approximate mathematical form for the repulsive potential can be utilized. We treat the charged impurity as a simple statically screened Coulombic center. Furthermore, it is assumed that electronic trapping into the bound state lying within the central core region can be represented by a three-dimensional quantum well. Consequently, the associated potential energy function $V(x)$ can be expressed as

$$
\begin{aligned}
& V(x)=V_{\max } \exp \left(-\frac{x-a_{0}}{\lambda_{d}}\right) \text { for } a_{0}<x, \\
& V(x)=0 \text { for } 0<x<a_{0},
\end{aligned}
$$

with $\lambda_{d}=\left\{\left[k_{B} T \epsilon\right] /\left(e^{2} n\right)\right\}^{1 / 2}$ the Debye screening length, $V_{\max }$ the potential barrier height, " $n$ " the free carrier density, $a_{0}$ the spatial extent of the trapping potential, and " $x$ " the radial distance from the central core. The above choice for the potential assumes that the average spacing between the defects is sufficiently large, and hence any coupling between neighboring sites can be ignored. The potential function given in Eq. (1) is not unique, and other mathematical forms [for instance: $V(x)=V_{\max } x \exp \left(-x / \lambda_{d}\right)$ ] would also yield a valid, symmetric three-dimensional repulsive trapping potential. Though more refined potentials could be used, the field-dependent trends obtained from Eq. (1) are expected to be generally accurate. As a result, Eq. (1) will be deemed to adequately serve our present purposes of demonstrating general trends in the field- dependent trapping behavior, and to bring out the qualitative features and underlying physical effects.

Variations in the trapping coefficients with increasing electric fields arise due to changes in the electronic distribution function which affects the associated tunneling process through the barrier structure. Thermionic emission of hot electrons over the potential barrier as a means of trap filling, however, is expected to be negligible in the present context for two reasons. First, the successful capture of such hot electrons will require a long sequence of sustained phonon emissions which is not very likely. Second, with a longer time duration, the probability of electronic tunneling out of the confining barrier will become quite substantial. Ignoring therefore the thermionic emission process, and using electronic tunneling as the primary means of capture, the following field dependence of the capture coefficient results:

$$
\operatorname{ratio}(F)=\frac{\int_{0}^{\hbar \omega} G(E) f(E, F) T(E) d E}{\int_{0}^{\hbar \omega} G(E) f(E, 0) T(E) d E},
$$

where $G(E)$ is the energy-dependent density of states function, $T(E)$ is the tunneling probability, and $f(E, F)$ is the electronic distribution function at a given electric field $F$. The upper limit of $\hbar \omega$ corresponds to the highest optical phonon energy, and denotes a cutoff above which energetic electrons are not expected to be captured. As already discussed, the probability of capture for such energetic electrons is low since they are more likely to undergo phonon absorption or to tunnel out of the barrier. The tunneling coefficient $T(E)$ can be evaluated using the WentzelKramers-Brillouin approximation which is fairly accurate at larger energies. The following analytical expression results:

$$
T(E)=\exp \left[-2 \int_{a_{0}}^{x_{M}} d x \sqrt{\frac{2 m^{*}}{\hbar^{2}}} \sqrt{V_{\max } \exp \left(-\frac{x}{\lambda_{d}}\right)-E},\right.
$$

where the upper limit $x_{m}=a_{0}-\lambda_{d} \ln \left\{E / V_{\max }\right\}$. For our choice of the screened potential, the above integration in Eq. (3) can be performed, to yield the following closed form solution:

$$
\begin{aligned}
T(E)= & \exp \left\{-\frac{\sqrt{32 m^{*}}}{\hbar} \lambda_{d}\left[\sqrt{V_{\max }-E}\right.\right. \\
& \left.\left.-\sqrt{E} \tan ^{-1}\left(\sqrt{\frac{V_{\max }-E}{E}}\right)\right]\right\} .
\end{aligned}
$$

Using Eq. (4) in Eq. (2), the field dependence of the capture coefficient can finally be worked out completely for a given Debye length and potential barrier value if the distribution function $f(E, F)$ were known. In the present scheme, we use the Monte Carlo procedure to numerically evaluate and determine $f(E, F)$. A simple, three valley model for electronic transport, as described in detail elsewhere, ${ }^{24}$ is used for the purpose with material values for GaAs. The appropriate electron-phonon and electronelectron scattering mechanisms have been incorporated into the simulation procedure based on static screening. 


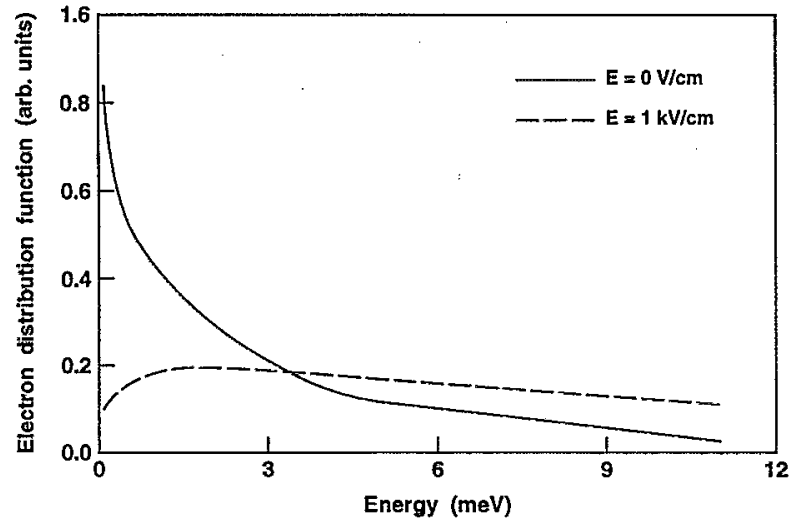

FIG. 1. Electron distribution functions at $77 \mathrm{~K}$ in GaAs obtained from Monte Carlo simulations. They correspond to electric field values of 0 and $1.0 \mathrm{kV} / \mathrm{cm}$.

The results of the Monte Carlo program yield the requisite electronic distribution function with the electric field as a variable parameter. Consequently, the ratio of Eq. (2) can be completely and accurately evaluated for any electric field value.

Finally, it must be pointed out that Eq. (2) used for the field-dependent electronic trapping in the present context differs from the expression used previously by Sacks and Milnes. ${ }^{9}$ The limits of integration do not extend to infinity since the capture of high energy electrons is very unlikely. Second, unlike their approach, tunneling through the repulsive barrier has explicitly been taken into account through a screened potential. Screening effects therefore, are naturally included. And lastly, the Monte Carlo evaluation technique used here yields a more accurate and exact evaluation of the field-dependent distribution function. Our calculations for $\mathrm{GaAs}$ will extend to much higher electric fields, and unlike previous results, the capture coefficient will be shown to decrease at the high field values.

\section{RESULTS AND DISCUSSION}

The Monte Carlo simulations were carried out for $\mathrm{GaAs}$ at $77 \mathrm{~K}$ to obtain the electronic distribution functions at various electric fields. The distribution is required for obtaining the field-dependent trapping ratio as given by Eq. (2). As in previous implementations, ${ }^{24} 10000$ particles were used, with a time step of $5 \mathrm{fs}$. The total simulation time was 25 ps which is adequate for obtaining all the steady-state estimators. The resulting distribution function at a temperature of $77 \mathrm{~K}$ is shown in Fig. 1 for the electric fields of 0 and $1 \mathrm{kV} / \mathrm{cm}$. In the absence of an electric field, the distribution is almost Maxwellian, while at the higher field value it exhibits a heated-drifted form.

Based on the output of Monte Carlo simulations, the field-dependent ratio for electronic trapping was computed next. The results obtained at an electronic concentration of $5 \times 10^{16} \mathrm{~cm}^{-3}$ and a temperature of $77 \mathrm{~K}$ are shown in Fig. 2. The curves correspond to three different values of the repulsive potential $V_{\max }$ chosen to be 50,100 , and 200 $\mathrm{meV}$, respectively, which are close to recently reported

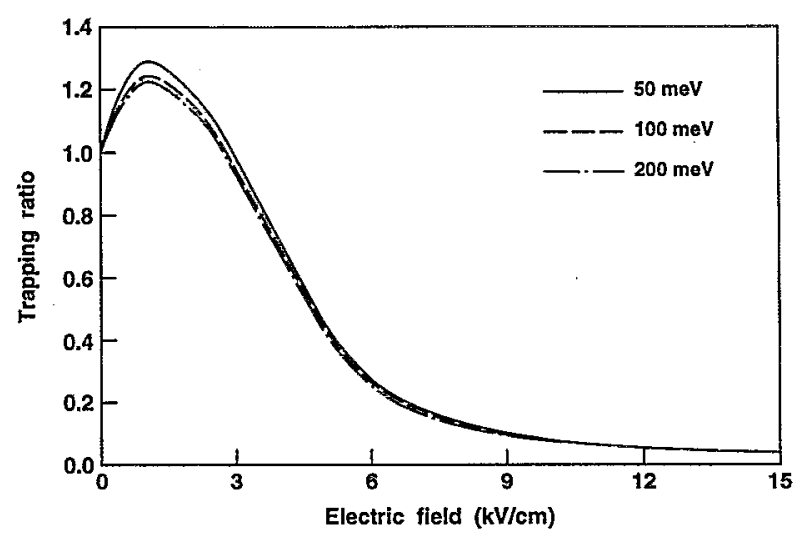

FIG. 2. Simulation results showing the electric field dependence of the trapping ratio at repulsive potentials of 50,100 , and $200 \mathrm{meV}$. A temperature of $77 \mathrm{~K}$ was used.

values. ${ }^{25}$ The general trend exhibited by the curves corresponds to an initial increase of the capture coefficient with electric field, followed by a monotonic reduction. Physically, such behavior can be understood by considering both intervalley transfer and the role of the maximum optical phonon energy. Initially at low fields, increases in the electric field lead to stronger heating of the gamma-valley electrons. This enhances the fraction of high energy electrons that are capable of tunneling through the repulsive barrier. Consequently, the trapping ratio is augmented. At higher electric fields beyond a critical limit however, the capture probability decreases as the mean energy of the electronic population begins to exceed that of the dominant optical phonons. As a result, a smaller fraction of the electrons have the chance of eventually being captured through a phonon cascade. Hot phonon effects, though not considered here, would work to further curtail the capture probability by enhancing the phonon absorption process.

Besides the above effect associated with the gammavalley electrons, electronic intervalley transfer at high electric fields contributes to the general trend. The increased effective mass associated with field-enhanced satellite valley occupancy, reduces the tunneling probability as given in Eq. (4). Such an intervalley effect will occur in all direct band gap materials. In indirect semiconductors such as silicon, however, an interesting orientational dependence of the critical field is expected. This should occur since the constant energy surfaces are ellipsoids rather than spheres, and the effective mass is directionally dependent. Consequently, depending on the applied field direction relative to the $\langle 100\rangle$ symmetry axes, the effective mass and hence the tunneling coefficient would vary. Finally, it may be noted that in common indirect semiconductors such as silicon or diamond, the value of the critical electric field beyond which the coefficient begins to decrease should be higher than in GaAs. This follows from the fact that the electronic effective mass in these semiconductors is substantially larger than in GaAs.

Finally, we examine the consequences arising from variations of free carrier screening. Changes in the carrier 


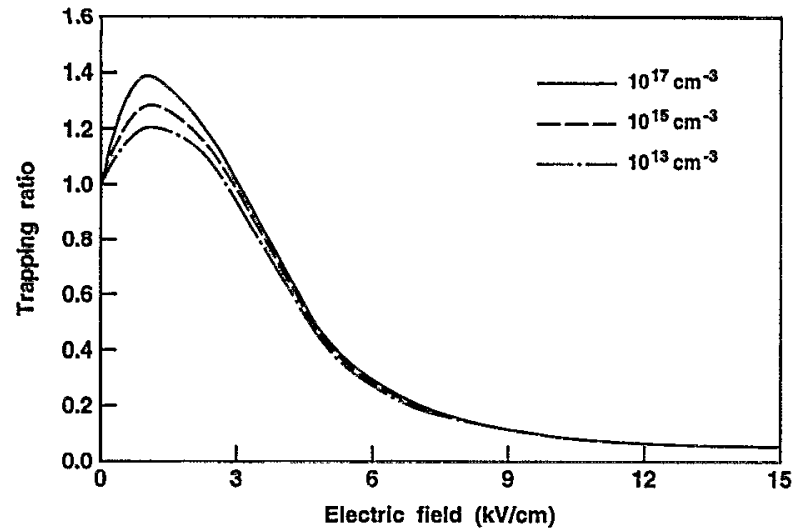

FIG. 3. Simulation results demonstrating effects of screening on the field dependence of the trapping coefficient. The curves correspond to electron densities of $10^{13}, 10^{15}$, and $10^{17} \mathrm{~cm}^{-3}$.

density affect the Debye length, and hence the tunneling coefficient directly through Eq. (4). The electronic distribution function is also modified to a small degree. Simulation results of the field-dependent curves for electronic concentrations of $10^{13}, 10^{15}$, and $10^{17} \mathrm{~cm}^{-3}$ are shown in Fig. 3. The trapping ratio from the plot is seen to have the smallest increase at low fields for the lowest density, which corresponds to the highest screening length. This is not surprising since the longer the screening length, the lower the tunneling coefficient, and hence the larger would be the electric field necessary for electrons to cross the repulsive barrier. However, a larger field value implies that effects associated with intervalley transfer and the optical phonon limiting energy would play a greater role. Consequently, increases in the trapping coefficient relative to the zero field value would not be quite as pronounced at lower densities, as is indeed demonstrated through our results. Though the simulation results shown in Fig. 3 were obtained at $77 \mathrm{~K}$, the screening effect can be expected to become stronger at higher temperatures. At $300 \mathrm{~K}$, for example, the Debye length would be larger for the same carrier density, and hence the density dependence of the trapping ratio would be more pronounced.

Finally, based on the numerical results obtained here, one can examine the possible impact on domain formation and the stability of compensated semiconductors containing deep-level impurities. Though a detailed analysis requires a self-consistent numerical solution of the semiconductor transport equations, a rough estimate of the stability can be obtained through a perturbative smallsignal analysis. To do so, we start from the set of onedimensional equations, neglect diffusion, and assume a uniform density of shallow donors $N_{D}^{+}$. We denote $N_{T}$ as being the deep-level trap density, $N_{T}^{-}(x, t)$ the singly charged trap density, $N_{T}^{-}(x, t)$ the doubly charged deep level profile, $n(x, t)$ the free-carrier concentration, $e_{n}$ the emission constant for the singly charged traps, $e_{n 1}$ the emission constant for doubly charged traps, $c_{n}$ the capture constant into a neutral trap state, and $c_{n 1}$ the capture coefficient for the singly ionized level. In the present case, we know from the preceding Monte Carlo calculations that $c_{n 1}$ is field dependent. Furthermore, the slope of $c_{n 1}$ is positive up to a certain value of the electric field, and then becomes negative. The resulting set of equations in terms of the above notation then is given as

$$
\begin{aligned}
& \frac{\partial F(x, t)}{\partial x}=\frac{q}{\epsilon}\left[N_{D}^{+}-n(x, t)-N_{T}^{-}(x, t)-2 N_{T}^{-}(x, t)\right], \\
& \begin{aligned}
\frac{\partial N_{T}^{-}(x, t)}{\partial t}= & c_{n} n(x, t)\left[N_{T}-N_{T}^{-}(x, t)-N_{T}^{-}(x, t)\right] \\
& -e_{n} N_{T}^{-}(x, t)-c_{n 1} n(x, t) N_{T}^{-}(x, t) \\
& +e_{n 1} N_{T}^{-}(x, t),
\end{aligned}
\end{aligned}
$$

$\frac{\partial N_{T}^{-}(x, t)}{\partial t}=c_{n 1} n(x, t) N_{T}^{-}(x, t)-e_{n 1} N_{T}^{--}(x, t)$,

$$
\begin{aligned}
\frac{\partial n(x, t)}{\partial t} & -\frac{\partial[v n(x, t)]}{\partial x} \\
= & e_{n} N_{T}^{-}(x, t)-e_{n 1} N_{T}^{-}(x, t)-c_{n} n(x, t)\left[N_{T}-N_{T}^{-}(x, t)\right. \\
& \left.-N_{T}^{-}(x, t)\right]-c_{n 1} n(x, t) N_{T}^{-}(x, t) .
\end{aligned}
$$

For the equation set (5), the perturbative solutions can be written as follows:

$$
\begin{aligned}
& F(x, t)=F_{0}+\delta F \exp [i(k x-\omega t)], \\
& n(x, t)=n_{0}+\delta n \exp [i(k x-\omega t)], \\
& N_{T}^{-}(x, t)=N_{T 0}^{-}-\delta N_{T}^{-} \exp [i(k x-\omega t)], \\
& N_{T}^{-}-(x, t)=N_{T 0}^{-}-\delta N_{T}^{-}-\exp [i(k x-\omega t)] .
\end{aligned}
$$

Using the solution set (6) in Eqs. (5) then yields the desired dispersion relation. In this case careful algebraic manipulation yields the following cubic expression for $\omega$ :

$$
\begin{aligned}
& \omega^{2}\left(\frac{q}{\epsilon} A_{1}+i k A_{2}+k^{2} v\right) \\
& -\omega\left(\frac{q n k \mu}{\epsilon} B_{1}-\frac{i q n N_{T}^{-}}{\epsilon} \frac{\partial C_{n 1}}{\partial F} B_{2}-\frac{3 q k v}{\epsilon}+k B_{3}-i k^{2} B_{4}\right) \\
& \quad+k \omega^{3}-\frac{q n i k \mu}{\epsilon} C_{1}-k^{2} v C_{2}+\frac{q n N_{T}^{-}}{\epsilon} \frac{\partial C_{n 1}}{\partial F}\left(C_{3}+i k C_{4}\right) \\
& \quad=0
\end{aligned}
$$

where $A_{1}=i n k \mu+2 n N_{T}^{-}\left\{\partial C_{n 1} / \partial F\right\} ; \quad A_{2}=c_{n} n+e_{n}+c_{n 1} n$ $+c_{n 1} N_{T}^{-}+e_{n 1}+c_{n}\left\{N_{T}-N_{T}^{-}-N_{T}^{-}\right\} ; \quad B_{1}=c_{n} n+e_{n}+c_{n 1} n$ $+e_{n 1}+c_{n}\left(N_{T}-N_{T}^{-}-N_{T}^{-}\right)+c_{n 1} N_{T}^{-} ; \quad B_{2}=2 c_{n 1} N_{T}^{-}+2 c_{n}$ $\times\left(N_{T}-N_{T}^{-}-N_{T}^{-}\right)-2 c_{n} n ; \quad B_{3}=c_{n}\left(N_{T}-N_{T}^{\bar{T}}-N_{T}^{-}\right)$ $\left(e_{n 1}+2 c_{n 1} n\right)+C_{n 1} N_{T}^{-}\left(2 e_{n}+n c_{n}\right)+n^{2} c_{n} c_{n 1}+e_{n 1}\left(c_{n} n+e_{n}\right) ;$ $B_{4}=v\left(n c_{n 1}+n c_{n}+e_{n}+e_{n 1}\right) ; \quad C_{1}=e_{n 1}\left(c_{n} n+e_{n}+c_{n}\left\{N_{T}\right.\right.$ $\left.\left.-N_{T}^{\bar{T}}-N_{T}^{-}-\right\}\right)+c_{n 1} N_{T}^{\bar{T}}\left(c_{n} n+2 e_{n}+c_{n 1} n\right)+c_{n 1} n\left(c_{n}\left\{N_{T}\right.\right.$ $\left.\left.-N_{T}^{-}-N_{T}^{-}\right\}+c_{n} n\right) ; \quad C_{2}=n c_{n}\left(n c_{n 1}+e_{n 1}\right)+e_{n} e_{n 1} ; \quad$ and $C_{3}=2 c_{n}\left\{N_{T}-N_{T}^{-}-N_{T}^{-}\right\}\left(c_{n} n+c_{n 1} n+e_{n} e_{n 1}\right)+\left(2 c_{n} n\right.$ $\left.+2 e_{n}+c_{n 1} n\right)\left(e_{n 1}+c_{n} n\right)+c_{n 1} N_{T}^{-}\left(3 c_{n} n+2 c_{n 1} n+2 e_{n}-e_{n 1}\right)$, and $C_{4}=v\left(2 e_{n 1}-c_{n} n-2 c_{n 1} n\right)$. In general then, the full dis- 
persion curve can be obtained numerically for a given set of input parameters and material characteristics.

The above dispersion relation is rather involved, and hence is not easily amenable to a stability analysis. However, it is possible to make reasonable predictions of instabilities in the low frequency regime by ignoring higher order terms in $\omega$. Besides the mathematical simplicity, a low frequency analysis is important from a practical standpoint since such low frequency oscillations and instabilities have actually been observed in experiments. ${ }^{2,3}$ In more recent experimental work on Cu-doped GaAs photoconductive switches, Franz-Keldysh measurements of the internal electric field have yielded direct observations of nearly stationary waves in millimeter length samples. ${ }^{26}$ Similar reports for dc biased semiconductor switches have also been described in the literature. ${ }^{27}$ So proceeding with the low frequency stability analysis, we ignore all of the nonlinear terms in $\omega$, and rewrite Eq. (7) in the following form:

$$
\begin{aligned}
\omega\left(P_{1}-i P_{2} \frac{\partial C_{n 1}}{\partial F}-i P_{3}\right)= & -i Q_{1}-Q_{2}+Q_{3} \frac{\partial C_{n 1}}{\partial F} \\
& +i Q_{4} \frac{\partial C_{n 1}}{\partial F}
\end{aligned}
$$

where the coefficients $P_{i}$ and $Q_{i}$ can be expressed in terms of the various $B_{i}$ and $C_{i}$ terms already defined. An expression for the imaginary part of $\omega$ can then be easily obtained from Eq. (8). For instability, one requires that the imaginary part be positive and hence the following must hold:

$$
\begin{aligned}
& -P_{1} Q_{1}+\left(P_{1} Q_{4}-P_{2} Q_{2}+P_{3} Q_{3}\right) \frac{\partial C_{n 1}}{\partial F}+P_{2} Q_{3}\left(\frac{\partial C_{n 1}}{\partial F}\right)^{2} \\
& -P_{3} Q_{2}>0
\end{aligned}
$$

Clearly if $C_{n 1}$ were assumed to be field independent, two of the above four terms would vanish and it would be difficult to create conditions for an instability. However, in situations where $C_{n 1}$ was increasing with the electric field, it would be easier for the imaginary component of $\omega$ to be positive. Hence, the potential for unstable growth of a low frequency charge wave exists for fields below a critical threshold. Furthermore, since this mechanism primarily requires the presence of deep defect levels alone, and is not associated with intervalley transfer, it could occur in both direct and indirect semiconductors. In fact, for materials such as silicon or diamond for which the larger electronic mass is much larger than in GaAs, the phenomena of such a trap-related instability should extend to higher electric fields. Experimentally it should be possible to detect the presence of such instabilities through electro-optic sampling techniques or by measurements of the timedependent currents. Finally, we mention that the above analysis can easily be extended to multilevel defects in compensated semiconductors. However, in such a situation, one expects the defect state having the lowest repulsive potential to play the most dominant role.

\section{CONCLUSIONS}

In summary, we have performed Monte Carlo simulations to obtain the field-dependent behavior of electronic trapping at repulsive potentials. Such repulsive centers would be associated with deep level impurities having multiply charged states. The results are based on a microscopic approach, which accurately takes account of the internal dynamics within the electronic system. As a consequence, the temperature effects, electric field heating, electronic screening, and the tunneling mechanisms are adequately included. Our results reveal a field-dependent structure in the electronic capture coefficient. $U p$ to a critical field value, the capture coefficient increases relative to its zero field value. At even higher fields, the slope turns out to be negative. We have also demonstrated through our calculations that the relative field dependence is a function of free carrier screening.

Based on our Monte Carlo results, we have explored the stability of compensated semiconductors containing such repulsive centers. Our analysis clearly indicates an enhanced potential for low frequency charge waves within such semiconductor samples. This conclusion is in keeping with available experimental data on semi-insulating GaAs. In particular, the electrical characteristics of laser activated $\mathrm{Cu}: \mathrm{Si}: \mathrm{GaAs}$ high-power photoconductive switches have displayed such unstable behavior. ${ }^{20}$ Finally, we would like to note that besides the field-dependent trapping discussed here, other mechanisms such as nonlocal impact ionization, two-stream instabilities, and domain assisted detrapping would also play a role in current oscillations and unstable behavior. However, the other processes would require high fields and/or the presence of a bipolar plasma. The mechanism discussed here, on the other hand, would contribute even at low electric fields without the presence of holes.

\section{ACKNOWLEDGMENTS}

The authors acknowledge F. Peterkin (Old Dominion University) and M. Kushner (University of Illinois) for helpful discussions. The work was supported by BMDO and managed by ONR. The program monitor is Gabriel Roy. Partial support from a DOE grant through the Lawrence Livermore National Laboratory is also acknowledged.

${ }^{1}$ W. Shockley and W. T. Read, Phys. Rev. 87, 835 (1952); R. N. Hall, ibid. 87, 387 (1952).

${ }^{2}$ For example, S. W. Teitsworth, Appl. Phys. A 48, 127 (1989); K. Aoki and Y. Yamamoto, ibid. 48, 111 (1989).

${ }^{3}$ E. Scholl, Solid State Electron. 32, 1129 (1989); G. N. Maracas, W. Porod, D. A. Johnson, and D. K. Ferry, Physica B 134, 276 (1985).

${ }^{4}$ P. J. Price, in Fluctuation Phenomena in Solids, edited by R. E. Burgess (Academic, New York, 1965); A. Van der Ziel, in Noise Source, Characterization, and Measurement (Prentice-Hall, Englewood Cliffs, NJ, 1970).

${ }^{5}$ L. Reggiani, P. Lugli, and V. V. Mitin, Phys. Rev. Lett. 60, 736 (1988).

${ }^{6}$ M. A. Mark and P. Lambert, in Current Injection in Solids (Academic, New York, 1970).

${ }^{7}$ B. L. Belmont and M. S. Shur, J. Phys. D: Appl. Phys. 6, 842 (1973); B. L. Belmont and M. S. Shur, Sov. Phys. Semicond. 7, 50 (1973).

${ }^{8}$ B. K. Ridley, Br. J. Appl. Phys. 17, 595 (1966) 
${ }^{9}$ H. K. Sacks and A. G. Milnes, Int. J. Electron. 28, 565 (1970); 30, 49 (1971).

${ }^{10}$ E. E. Godik, Y. A. Kuritsyn, and V. P. Sinis, Sov. Phys. Second. 12, 203 (1978).

${ }^{11}$ R. P. Joshi, J. Appl. Phys. 74, 1810 (1993).

${ }^{12}$ M. Lax, Phys. Rev. 119, 1502 (1960).

${ }^{13}$ S. H. Koenig, Phys. Rev. 110, 988 (1958).

${ }^{14}$ E. F. Smith and P. T. Landsberg, J. Phys. Chem. Solids 27, 1727 (1966); W. Pickin, Phys. Status Solidi B 97, 431 (1980).

${ }^{15}$ V. N. Abakumov, V. I. Perel, and I. N. Yassievich, Sov. Phys. Semicond. 12, 1 (1978); V. V. Akulinichev and I. N. Yassievich, ibid. 16, 159 (1982).

${ }^{16}$ L. Reggiani, L. Varani, V. V. Mitin, and C. M. Van Vliet, Phys. Rev. Lett. 63, 1094 (1989); L. Reggiani and V. V. Mitin, Risivta Nuovo Cimento 12, 1 (1989).

${ }^{17}$ For example, H. Shichijo and K. Hess, Phys. Rev. B 23, 793 (1981).

${ }^{18}$ R. L. Kamocsai and W. Porod, J. Appl. Phys. 69, 2264 (1991), and references therin.

${ }^{19}$ For example, W. W. Tyler, and H. H. Woodbury, Phys. Rev, 96, 874
(1954); A. G. Milnes, in Deep Impurities in Semiconductors (Wiley, New York, 1973).

${ }^{20}$ K. H. Schoenbach, in High-Power Optically Activated Solid State Switches, edited by A. Roden and F. Zutavern (Artech House, Boston, 1993).

${ }^{21}$ D. Krokel, D. Grischkowsky, and M. B. Ketchen, Appl. Phys. Lett. 54, 1046 (1989); N. Katzenellenbogen and D. Grischkowsky, Appl. Phys. Lett. 58, 222 (1991).

${ }^{22}$ B. K. Ridley and F. A. El-Ela, J. Phys.: Condens. Matter 1, 7021 (1989); B. K. Ridley, ibid. 2, 2941 (1990).

${ }^{23}$ N. Bannov, V. Gruzinskis, A. Reklaitis, and V. Ryzhij, Solid State Electron. 29, 1207 (1986).

${ }^{24}$ R. P. Joshi, R. O. Grondin, and D. K. Ferry, Phys. Rev. B 42, 5685 (1990); S. Chamoun, R. P. Joshi, R. O. Grondin, K. Meyer, and G. Mourou, J. Appl. Phys. 66, 236 (1989).

${ }^{25}$ M. Schulz, J. Appl. Phys. 74, 2649 (1993).

${ }^{26}$ K. H. Schoenbach, J. S. Kenney, F. E. Peterkin, and R. J. Allen, Appl. Phys. Lett. 63, 2100 (1993), and references therein.

${ }^{27}$ J. C. Adams, C. D. Kapps, R. A. Falk, and S. Ferrier, Appl. Phys. Lett. 63, 633 (1993). 